\title{
Pharmacovigilance - A Medication Safety Perspective
}

\author{
Syed Saad Hussain*
}

School of Pharmacy, Iqra University, Karachi, Pakistan.

The aim of pharmacovigilance (PV) is to evaluate the risk of adverse events of medication being administered to patients keeping in mind that no medication is completely safe [1]. Goal of therapeutics is to improve patient quality of life with minimizing risk and harmful effects associated with it. Medication safety is the process which optimizes the use of medicines and prevents from harm. Medicines are generally safe but as chemical in nature and xenobiotics to living organism are associated with unknown and known risk. Previously, drug safety issues were not discussed galanty due to false adversity consideration [2], but with time and awareness this has changed with highlighting the importance and necessity of pharmacovigilance. WHO classifies pharmacovigilance as the science and process that is associated with detection, assessment, understanding and prevention of adverse effects or any other drug related problem [3]. Moreover, we should consider that the medications to be administered to patient must be assessed by the science of pharmacovigilance. It is the activity that majorly involve monitoring the effect of any medication during its life cycle starting from manufacturing to the patient's use [4].

Pharmacovigilance has come into effectiveness after the thalidomide disaster which affected as many as a generation of population who were exposed during their fetal development. This led to establishment of WHO Collaborating Centre for International Drug Monitoring, Uppsala Monitoring Centre, (UMC), in Sweden [5]. Medication safety is a very important aspect of therapeutics that enhances the effectiveness and optimization of patient care. Any harmful effect that adversely effects a patient is now considered to come under pharmacovigilance scope [6]. Regulatory authorities now consider post surveillance monitoring an important process to assess the impact of medication on a particular population as clinical trials lack the holistic approach due to certain limitation in exposure to all patients i.e. elderly, pregnant, etc. Pharmacovigilance processes are capable of generating signals by assessment of collected data through data mining. It is the first step that exposes the potential additional risk to those identified in clinical trials and further ascertains the benefit - risk ratio for the use of medications [7].

Demographic characteristics play an important role in pharmacodynamic and pharmacokinetic profile of any pharmaceutical product [8]. Pharmacovigilance for countries

*Address correspondence to this author at the School of Pharmacy, Iqra

University, Karachi, Pakistan. Email: saad.hussain96@outlook.com where generic medicines are more profoundly found is more crucial as clinical trials are majorly run in those countries where initial approval is being sought for or world leaders in health system. Countries like Pakistan where there is very limited exposure to population in terms of phase 1 and phase 2 clinical trials, pharmacovigilance or passive surveillance is the only process which can assess the robust pharmacodynamic properties and safety in relation to specific pharmacokinetic parameters of a specific population.

The other aspect that also comes under the scope of pharmacovigilance are adverse drug events that are related to the administration, dispensing or other aspects rather than adverse drug reactions. Robust health care systems are developed by identifying the loop holes in the process that can increase the risk of harmful effect on the patient rather than curative effect. Identification of loop holes are based on the data collection of any probable near miss or occurrence of any adverse drug event. Pharmacovigilance is the science that collects the data for analysis. Analysis leads to signal detection regarding any probable cause of harmful event. Signal generation further paves the way for research and further communication of safety aspect. Lack of efficacy cases can also be identified through the activities of pharmacovigilance [5] which can be a basis of choice of first line therapy and antimicrobial resistance.

In Pakistan, the physicians were knowledgeable about medication safety and ADR reporting. However they were not informed of the local pharmacovigilance centers in the country [9]. The future healthcare professionals, particularly the pharmacist were motivated to practice pharmacovigilance and participate in pharmacovigilance activities [10].

PV activities commonly employed include collection and collation of individual case safety reports (ICSRs), action after signal detection safety information vigilance and training of healthcare associated personnel [11]. In some regions, PV activities are evolving as a regulatory activity [12]. These activities are considered as integral part of medication safety process. Increasing the practice of pharmacovigilance will lead to better medication safety and optimizing the use of therapeutics. We should be more considerate in implementing the science of pharmacovigilance from reporting of drug related events to signal detection to communication for betterment of all societies. 


\section{CONFLICT OF INTEREST}

Declared none.

\section{ACKNOWLEDGEMENTS}

Declared none.

\section{REFERENCES}

[1] Pitts PJ, Louet HL, Moride Y, Conti RM. 21st century pharmacovigilance: Efforts, roles, and responsibilities. Lancet Oncol 2016; 17(11): e486-92. DOI: 10.1016/S1470-2045(16)30312-6

[2] Hussain R, Hassali MA. Current status and future prospects of pharmacovigilance in Pakistan. J Pharm Policy Pract 2019; 12: 14. DOI: $10.1186 / \mathrm{s} 40545-019-0178-\mathrm{x}$

[3] Pharmacovigilance - Regulation and Prequalification. 2021; Available at: https://www.who.int/teams/regulation-prequalification/pharmacovigilance. Accessed on [13 February 2021].

[4] European Commission. Monitoring safety of medicines for patient-Pharmacovigilance activities related to medicines for human use in the EU. 2016; Available at: https://ec.europa.eu/health/sites/default/files/files/pharmacovigilance/pharmacovigilance-report-2012-2014.pdf Accessed on [13 February 2021].

[5] Huff-Rousselle M, Simooya O, Kabwe V. Pharmacovigilance and new essential drugs in Africa: Zambia draws lessons from its own experiences and beyond. Global Public Health 2007; 2(2): 184-203. DOI: 10.1080/17441690601063299
[6] Toklu HZ, MensahE. Why do we need pharmacists in pharmacovigilance systems? Online J Public Health Inform 2016; 8(2): e193. DOI: $10.5210 /$ ojphi.v8i2.6802

[7] Waller P, Harrison-Woolrych M. An Introduction to Pharmacovigilance. $2^{\text {nd }}$ ed. USA: Wiley-Blackwell 2017. DOI: 10.1002/9781119289777

[8] Gong IY, Kim RB. Importance of pharmacokinetic profile and variability as determinants of dose and response to dabigatran, rivaroxaban, and apixaban. Can J Cardiol 2013; 29(7): S24-33. DOI: 10.1016/j.cjca.2013.04.002

[9] Hussain R, Hassali MA, ur Rehman A, Muneswarao J, Hashmi F. Physicians' understanding and practices of pharmacovigilance: qualitative experience from a lower middle-income country. Int J Environ Res Public Health 2020; 17(7): 2209. DOI: 10.3390/ijerph17072209

[10] Shakeel S, Iffat W, Anjum F, Bushra R, Ibrahim S, Shafiq S. Emerging need of pharmacovigilance: Perspectives of future pharmacist in Pakistan. Int J Pharm Teach Pract 2014; 5(2): $1-6$.

[11] Suwankesawong W, Dhippayom T, Tan-Koi WC, Kongkaew C. Pharmacovigilance activities in ASEAN countries. Pharmacoepidemiol Drug Saf 2016; 25(9): 1061-9. DOI: $10.1002 /$ pds. 4023

[12] World Health Organization. The importance of pharmacovigilance. 2002; Available at: https://apps.who.int/iris/bitstream/handle/10665/42493/a75646.pdf. Accessed on [13 February 2021]. 